\title{
DUALITY THEOREMS FOR CONVEX FUNCTIONS
}

\author{
BY R. T. ROCKAFELLAR ${ }^{1}$
}

Communicated by A. M. Gleason, September 19, 1963

Let $F$ be a finite-dimensional real vector space. A proper convex function on $F$ is an everywhere-defined function $f$ such that $-\infty<f(x)$ for all $x, f(x)<\infty$ for at least one $x$, and

$$
f\left(\lambda x_{1}+(1-\lambda) x_{2}\right) \leqq \lambda f\left(x_{1}\right)+(1-\lambda) f\left(x_{2}\right)
$$

for all $x_{1}$ and $x_{2}$ when $0<\lambda<1$. Its effective domain is the convex set $\operatorname{dom} f=\{x \mid f(x)<\infty\}$. Its conjugate $[2 ; 3 ; 6 ; 7]$ is the function $f^{*}$ defined by

$$
f^{*}\left(x^{*}\right)=\sup \left\{\left(x, x^{*}\right)-f(x) \mid x \in F\right\} \quad \text { for each } x^{*} \in F^{*},
$$

where $F^{*}$ is the space of linear functionals on $F$. The conjugate function is proper convex on $F^{*}$, and is always lower semi-continuous. If $f$ itself is l.s.c., then $f$ coincides with the conjugate $f^{* *}$ of $f^{*}$ (where $F^{* *}$ is identified with $F$ ). These facts and definitions have obvious analogs for concave functions, with "inf" replacing "sup" in (1).

Suppose $f$ is l.s.c. proper convex on $F$ and $g$ is u.s.c. proper concave on $F$. If

$$
\text { ri }(\operatorname{dom} f) \cap \operatorname{ri}(\operatorname{dom} g) \neq \varnothing,
$$

where ri $C$ denotes the relative interior of a convex set $C$, then

$$
\inf \{f(x)-g(x) \mid x \in F\}=\max \left\{g^{*}\left(x^{*}\right)-f^{*}\left(x^{*}\right) \mid x^{*} \in F^{*}\right\} .
$$

This was proved by Fenchel [3, p. 108] (reproduced in [5, p. 228]). The purpose of this note is to announce the following more general fact.

Theorem 1. Let $F$ and $G$ be finite-dimensional partially-ordered real vector spaces in which the nonnegative cones $P(F)$ and $P(G)$ are polyhedral. Let $A$ be a linear transformation from $F$ to $G$. Let $f$ be a proper convex function on $F$ and let $g$ be a proper concave function on $G$. If there exists at least one $x \in \operatorname{ri}(\operatorname{dom} f)$ such that $x \geqq 0$ and $A x \geqq y$ for some $y \in \mathrm{ri}(\operatorname{dom} g)$, then

$$
\text { inf } \begin{aligned}
\{f(x)-g(y) \mid x & \geqq 0, A x \geqq y\} \\
& =\max \left\{g^{*}\left(y^{*}\right)-f^{*}\left(x^{*}\right) \mid y^{*} \geqq 0, A^{*} y^{*} \leqq x^{*}\right\},
\end{aligned}
$$

where $A^{*}$ is the adjoint of $A$.

1 The material in this note stems from the author's recent doctoral dissertation at Harvard. Support was provided under grant AF-AFOSR-62-348 at the Computation Center, Massachusetts Institute of Technology. 
The partial-orderings are, of course, assumed to be compatible with the vector structure. The orderings in $F^{*}$ and $G^{*}$ are dual to those in $F$ and $G$, i.e. $P\left(F^{*}\right)$ consists of the $x^{*}$ such that $\left(x, x^{*}\right) \geqq 0$ whenever $x \geqq 0$, etc.

In particular, any $F$ and $G$ can be supplied with the degenerate partial-orderings in which $P(F)=F$ and $P(G)=\{0\}$, so that $P\left(F^{*}\right)$ $=\{0\}$ and $P\left(G^{*}\right)=G^{*}$. If Theorem 1 is then invoked, one obtains

Corollary 1. Assume the notation of Theorem 1, but omit the partialordering of $F$ and $G$. If $A x \in \operatorname{ri}(\operatorname{dom} g)$ for at least one $x \in \operatorname{ri}(\operatorname{dom} f)$, then

$\left(2^{\prime}\right) \inf \{f(x)-g(A x) \mid x \in F\}=\max \left\{g^{*}\left(y^{*}\right)-f^{*}\left(A^{*} y^{*}\right) \mid y^{*} \in G^{*}\right\}$.

When $F=G$ and $A=I$, Corollary 1 furnishes a slightly generalized version of Fenchel's theorem not requiring semi-continuity.

Another new result is the following.

CoRollary 2. Assume the notation of Theorem 1, and suppose also that $\operatorname{dom} f, \operatorname{dom} f^{*}, \operatorname{dom} g$ and $\operatorname{dom} g^{*}$ are all linear manifolds. If any one of the following is true,

(a) inf $\{f(x)-g(y) \mid x \geqq 0, A x \geqq y\}$ is finite,

(b) $\sup \left\{g^{*}\left(y^{*}\right)-f^{*}\left(x^{*}\right) \mid y^{*} \geqq 0, A^{*} y^{*} \leqq x^{*}\right\}$ is finite,

(c) $\{\langle x, y\rangle \mid 0 \leqq x \in \operatorname{dom} f, A x \geqq y \in \operatorname{dom} g\} \neq \varnothing$ and

$$
\left\{\left\langle y^{*}, x^{*}\right\rangle \mid 0 \leqq y^{*} \in \operatorname{dom} g^{*}, A^{*} y^{*} \leqq x^{*} \in \operatorname{dom} f^{*}\right\} \neq \varnothing,
$$

then all three are true. Moreover, then the "inf" and "sup" are equal and both are attained.

This corollary is deduced from Theorem 1 and its dual (in which the roles of the starred and unstarred elements are reversed), using the trivial fact that ri $C=C$ when $C$ is a linear manifold. The appropriate semi-continuity of $f$ and $g$, which one needs in order that $f^{* *}=f$ and $g^{* *}=g$ in the dual of Theorem 1 , is also a consequence of the hypothesis, because a convex or concave function is actually continuous on any relatively open set where it is finite-valued.

Fix any $b^{*} \in F^{*}$ and $c \in G$. Let $f(x)=\left(x, b^{*}\right)$. Let $g(y)=0$ if $y=c$ and $g(y)=-\infty$ if $y \neq c$. Then $f^{*}\left(x^{*}\right)=0$ if $x^{*}=b^{*}, f^{*}\left(x^{*}\right)=\infty$ if $x^{*} \neq b^{*}$, and $g^{*}\left(y^{*}\right)=\left(c, y^{*}\right)$. In this situation, Corollary 2 yields the important existence and duality theorems of Gale, Kuhn and Tucker for linear programs (see [4]). Many other convex programming results, both new and old, are also contained in the theorem and its corollaries. The common extremum value can be characterized as a minimax. 
Theorem 1 is proved by way of a simpler theorem of some interest in itself.

THEOREM 2. Let $h$ be a proper convex function on a finite-dimensional real vector space $E$ and let $K$ be a polyhedral convex cone in $E$. If ri(dom $h$ ) intersects $K$, then

$$
\inf \{h(z) \mid z \in K\}=-\min \left\{h^{*}\left(z^{*}\right) \mid z^{*} \in K^{*}\right\},
$$

where $K^{*}=\left\{z^{*} \in E^{*} \mid\left(z, z^{*}\right) \geqq 0\right.$ for all $\left.z \in K\right\}$.

An outline of the proof of Theorem 2 follows. One shows first that no generality is lost if $h$ is assumed 1.s.c. Then one observes that (3) holds whenever ri(dom $h)$ actually intersects ri $K$. This is obtained from Fenchel's theorem by taking $f(z)=h(z), g(z)=0$ if $z \in K$, $g(z)=-\infty$ if $z \in K$. The proof proceeds now by induction on the dimension of $K$. If $\operatorname{dim} K=0$, then ri $K=K$ trivially, so (3) is true. Assume next that (3) is true for cones of dimension less than $r$, and that $\operatorname{dim} K=r$. It may be supposed that $\operatorname{ri}(\operatorname{dom} h)$ does not intersect ri $K$, since the other case has been covered. A separation argument then produces a $z_{0}^{*} \in K^{*}$ such that $-z_{0}^{*} \in K^{*}$ and

$$
\left(z, z_{0}^{*}\right) \leqq 0 \quad \text { for all } z \in \operatorname{dom} h .
$$

Let $K_{0}=\left\{z \in K \mid\left(z, z_{0}^{*}\right)=0\right\}$. Then $K_{0}$ is a polyhedral convex cone, and $\operatorname{dim} K_{0}<r$. Hence by the induction hypothesis

$$
\inf \left\{h(z) \mid z \in K_{0}\right\}=-\min \left\{h^{*}\left(z^{*}\right) \mid z^{*} \in K_{0}^{*}\right\} .
$$

It is easy to see from the properties of $z_{0}^{*}$ that the left sides of (3) and (5) are the same. On the other hand, because $K$ is polyhedral,

$$
K_{0}^{*}=\left\{z^{*}-\lambda z_{0}^{*} \mid z^{*} \in K^{*}, \lambda \geqq 0\right\} .
$$

Moreover, (4) and definition (1) imply that $h^{*}\left(z^{*}-\lambda z_{0}^{*}\right) \geqq h^{*}\left(z^{*}\right)$ for all $z^{*} \in E^{*}$ and $\lambda \geqq 0$. Therefore the minimum of $h^{*}$ on $K_{0}^{*}$ can be achieved on $K^{*}$ itself, so that the right sides of (3) and (5) are equivalent, too.

Theorem 1 is deduced from Theorem 2 by choosing

$$
\begin{aligned}
& E=\{z=\langle x, y\rangle \mid x \in F, y \in G\}, \quad h(z)=f(x)-g(y), \\
& K=\{\langle x, y\rangle \mid x \geqq 0, A x \geqq y\} .
\end{aligned}
$$

\section{REFERENCES}

1. E. Eisenberg, Duality in homogeneous programming, Proc. Amer. Math. Soc. 12 (1961), 783-787.

2. W. Fenchel, On conjugate convex functions, Canad. J. Math. 1 (1949), 73-77. 
3. - Convex cones, sets and functions, multilith lecture notes, Princeton Univ., Princeton, N. J., 1953.

4. A. J. Goldman and A. W. Tucker, Theory of linear programming, pp. 53-98, Annals of Mathematics Studies no. 38, Princeton Univ. Press, Princeton, N. J., 1956.

5. S. Karlin, Mathematical methods and theory in games, programming and economics, Vol. I, Addison-Wesley, Reading, Mass., 1960.

6. J.-J. Moreau, Fonctions convexes en dualité, Faculté des Sciences de Montpellier, Séminaires de Mathématiques, 1962 (multigraph).

7. - Fonctions convexes duales et points proximaux dans un espace hilbertien, C.R. Acad. Sci. Paris 255 (1962), 2897-2899.

\section{UnIversity of Texas}

